\title{
Crack Growth Characteristics of Aluminum Alloys Dominated by the Mechanisms of Fatigue and Stress Corrosion Cracking
}

\author{
Takeshi Ogawa ${ }^{1, *}$, Shota Hasunuma ${ }^{1}$, Shunsuke Kato ${ }^{2}$, Shunpei Suzuki ${ }^{3}$, Yuta Nakamura ${ }^{4}$, \\ Satomi Mano ${ }^{5}$ and Kazuo Miyagawa ${ }^{5}$ \\ ${ }^{1}$ Department of Mechanical Engineering, Aoyama Gakuin University, Sagamihara 252-5258, Japan \\ ${ }^{2}$ All Nippon Airways Co., Ltd., Tokyo 105-7140, Japan \\ ${ }^{3}$ Obayashi Corporation, Tokyo 108-8502, Japan \\ ${ }^{4}$ Central Japan Railway Company, Nagoya 450-6101, Japan \\ ${ }^{5}$ Honda R\&D Co., Ltd., Haga 321-3393, Japan
}

\begin{abstract}
Three aluminum alloys in the T6 state $(6061,6082$, and 7075$)$ were subjected to static and cyclic loading conditions in humid and dry air environments and the corresponding crack growth characteristics were investigated. The dominant crack growth mechanisms, i.e., stress corrosion cracking (SCC) and fatigue crack growth (FCG), were identified. Three types of testing machines were used to apply the cyclic loading at high stress ratio and frequencies ranging from $0.02 \mathrm{~Hz}$ to $20 \mathrm{kHz}$. Crack growth mechanisms are discussed based on the traditional model proposed by McEvily and Wei. Various test results revealed that SCC is the dominant crack growth mechanism at $0.02 \mathrm{~Hz}$, whereas FCG is dominant at $20 \mathrm{~Hz}$ and $20 \mathrm{kHz}$. These results suggested that the crack growth behavior is controlled by either SCC or FCG with no mutual interaction. Very slow crack growth (rate: $<10^{-13} \mathrm{~m} /$ cycle) occurred during ultrasonic fatigue tests performed at $20 \mathrm{kHz}$. For each alloy and stress ratio considered, this slow growth occurred only in humid air and at low values of the stress intensity factor. Scanning electron microscopy observations suggested that, owing to the numerous cyclic loads, the very slow crack growth behavior in the humid environment occurs via peeling-off of the oxide film near the crack tip. [doi:10.2320/matertrans.Z-M2019845]
\end{abstract}

(Received April 26, 2019; Accepted July 31, 2019; Published October 25, 2019)

Keywords: stress corrosion cracking, fatigue crack growth, high stress ratio, ultrasonic fatigue test, aluminum alloy

\section{Introduction}

A hydrogen gas container is one of the most important components of a fuel cell electric vehicle (FCEV). The metal boss and/or liners of the containers are composed of aluminum alloys, which are subjected to both static loads (induced by the high-pressure gas) and cyclic loads (resulting from hydrogen charging and consumption). These loads lead to damage, i.e., stress corrosion cracking (SCC) and fatigue crack growth (FCG) of the alloys. The relevant ISO standard stipulates that hydrogen gas as a fuel may contain $<5 \mathrm{ppm}$ of water vapor. Hence, when the gas is charged at $15^{\circ} \mathrm{C}$ up to $70 \mathrm{MPa}$, a relative humidity $(R H)$ of $>85 \%$ is expected for a container placed in a low-temperature (e.g., $-7^{\circ} \mathrm{C}$ ) environment. ${ }^{1)}$ Therefore, the characteristics of SCC and FCG in humid air are critical for the design of containers.

Previous studies have reported that the SCC and FCG of aluminum alloys are sensitive to $R H$. The former is referred to as humid gas stress corrosion cracking (HG-SCC) and a method for its characterization has been standardized by HPIS E103. ${ }^{2)}$ Ito has suggested that the HG-SCC mechanism involves hydrogen dissolved from water vapor. ${ }^{1)}$ Furthermore, Kawagoishi et al. ${ }^{3)}$ reported that hydrogen leads to changes in the slip mechanism. According to Endo et al., when a small cyclic load is superimposed on a static load, the SCC growth rate is higher than that associated with static loading of high-strength steels ${ }^{4)}$ and aluminum alloys ${ }^{5)}$ in $\mathrm{NaCl}$ solution. This behavior is referred to as dynamic or cyclic SCC, where the passive film at the crack tip is successively broken by the cyclic load. Ogawa et al. have investigated the HG-SCC characteristics of aluminum alloys

*Corresponding author, E-mail: ogawa@me.aoyama.ac.jp via two types of SCC tests. One test included partial unloading at every $1 \mathrm{ks}$ in order to measure the crack length via the compliance method, while the other consisted of a bolt loading test performed under completely static conditions. ${ }^{6)}$ The results revealed that the crack growth rate of the former test is higher than that of the latter. When the cyclic component of the loading is increased, the crack growth mechanism may transition from SCC to FCG, as suggested by McEvily and Wei. ${ }^{7)}$ Investigations focused on the transition behavior associated with the HG-SCC mechanism of aluminum alloys exposed to humid air are, however, lacking.

Therefore, in the present study, crack growth characteristics are investigated for aluminum alloys subjected to cyclic loading (FCG test) and static loading (SCC test) in humid and dry air environments. Three cyclic frequency, $f$, values of $0.02 \mathrm{~Hz}, 20 \mathrm{~Hz}$, and $20 \mathrm{kHz}$ are employed during the FCG tests. The role of the FCG or SCC mechanisms is determined based on $f$ - and stress ratio $(R)$-induced $(R: 0.5,0.8$, and 0.9$)$ changes in the cyclic component of the loading.

\section{Materials and Experimental Procedures}

Three aluminum alloys $(6061,6082$, and 7075) in the T6 state were investigated in this study (see Tables 1 and 2 for the chemical compositions and the mechanical properties, respectively, of the alloys). Compact (CT) specimens (width, $W: 50.8 \mathrm{~mm}$, and thickness, $B: 10 \mathrm{~mm}$ ) were employed for the SCC test and FCG test at $0.02 \mathrm{~Hz}$. These tests were performed with a cantilever type testing machine developed in-house using oil hydraulic actuators. ${ }^{8)}$ The CT specimens ( $W: 25.4 \mathrm{~mm}$ and $B: 5 \mathrm{~mm}$ ), subjected to FCG tests performed at $20 \mathrm{~Hz}$ on an in-house oil hydraulic fatigue testing machine, 
Table 1 Chemical composition (mass \%) of each test material.

\begin{tabular}{ccccccccc}
\hline Alloy & $\mathrm{Si}$ & $\mathrm{Fe}$ & $\mathrm{Cu}$ & $\mathrm{Mn}$ & $\mathrm{Mg}$ & $\mathrm{Cr}$ & $\mathrm{Zn}$ & $\mathrm{Ti}$ \\
\hline 6061 & 0.6 & 0.23 & 0.25 & 0.01 & 0.99 & 0.19 & $<0.01$ & 0.03 \\
\hline 6082 & 1.0 & 0.23 & $<0.01$ & 0.71 & 0.89 & 0.14 & $<0.01$ & 0.03 \\
\hline 7075 & 0.1 & 0.15 & 1.6 & 0.03 & 2.5 & 0.19 & 5.6 & 0.02 \\
\hline
\end{tabular}

Table 2 Mechanical properties of test materials subjected to T6 heat treatment.

\begin{tabular}{cccc}
\hline Alloy & Tensile strength $(\mathrm{MPa})$ & Proof stress $(\mathrm{MPa})$ & Elongation (\%) \\
\hline 6061 & 330 & 290 & 18 \\
\hline 6082 & 353 & 315 & 17 \\
\hline 7075 & 591 & 508 & 15 \\
\hline
\end{tabular}

were smaller than those tested at $0.02 \mathrm{~Hz}$. The FCG tests at $20 \mathrm{kHz}$ were performed on an in-house ultrasonic fatigue testing machine using a specimen (see Fig. 1) designed with a desired resonant frequency of $20 \mathrm{kHz} .{ }^{9)}$ These specimens were cut in the T-L orientation, where the loading and the crack growth directions are transverse (T) and longitudinal (L), respectively. Intermittent loading was conducted by monitoring the specimen temperature with a radiation thermometer. The typical loading condition consisted of $900 \mathrm{~ms}$ loading with $100 \mathrm{~ms}$ dwell time.

The tests were performed at room temperature $\left(24-25^{\circ} \mathrm{C}\right)$ in humid $(R H>90 \%)$ and in dry $(R H<20 \%)$ air environments. The dry air, which was supplied by P4-EFA (IAC Co., Ltd.), can reduce the dew point to $-17^{\circ} \mathrm{C}$. The crack length, $a$, was monitored via the (i) unloading compliance method during the SCC and FCG tests conducted at 0.02 and $20 \mathrm{~Hz}$, (ii) periodic surface replicating technique during the FCG test performed at $20 \mathrm{kHz}$. For the SCC test, $a$ was monitored by applying a static load for $100 \mathrm{ks}$ with partial unloading of $R=0.5$ at every $1 \mathrm{ks}$. Subsequently, the static load was increased by a factor of 1.2 until crack growth occurred at a sufficiently high rate, as described by Ogawa et al. ${ }^{10)}$ For the FCG test at $0.02 \mathrm{~Hz}$, a cyclic load at $R=0.5,0.8$, and 0.9 was applied in a similar manner to that employed in the SCC test, i.e., the load was applied and then partially unloaded (see above). The FCG test at $20 \mathrm{~Hz}$ was performed in accordance with ASTM E647. After introducing a precrack at $R=0.1$, tests involving an increase in the stress intensity factor range $(\Delta K)$ were conducted at $R=0.5,0.8$, and 0.9 .
The corresponding maximum stress intensity factor, $K_{\max }$, was lower than that associated with the precracking condition. When the crack growth increment $(\Delta a)$ and the number of cycles $(N)$ were $<0.1 \mathrm{~mm}$ and $10^{6}$, respectively, the value of $\Delta K$ increased by a factor of 1.2. The cyclic crack growth rate, $\mathrm{d} a / \mathrm{d} N$, was calculated from the data obtained for $\Delta a>0.3 \mathrm{~mm}$ and the corresponding $N$. For the FCG test at $20 \mathrm{kHz}$, a precrack with a length of $\sim 1 \mathrm{~mm}$ was introduced at $\Delta K=5 \mathrm{MPa} \sqrt{\mathrm{m}}$ and $R=-1 . \Delta K$-increasing tests were then performed at $R=0.5,0.8$, and 0.9 (initial $\Delta K$ values were $0.5 \mathrm{MPa} \sqrt{\mathrm{m}}$ for $R=0.8$ and 0.9 , and $1.2 \mathrm{MPa} \sqrt{\mathrm{m}}$ for $R=$ $0.5)$. When $\Delta a$ and $N$ were $<0.01 \mathrm{~mm}$ and $10^{6}$ cycles, respectively, the value of $\Delta K$ increased by a factor of 1.2 . Once $\mathrm{d} a / \mathrm{d} N$ surpassed $10^{-12} \mathrm{~m} /$ cycle, the crack length was measured considerably more frequently (under the same loading condition) than at the lower $\mathrm{d} a / \mathrm{d} N$ values.

\section{Results and Discussion}

\subsection{Crack growth rate}

Figure 2 shows the relationships between $\mathrm{d} a / \mathrm{d} N$ and $\Delta K$ at $R=0.8$ and 0.9 . When $\mathrm{d} a / \mathrm{d} N=10^{-9}-10^{-8} \mathrm{~m} /$ cycle, similar relationships are obtained for the alloys tested except the conditions at $20 \mathrm{kHz}$ and $\mathrm{RH}>90 \%$ as well as at $0.02 \mathrm{~Hz}$ for both environments, which are excluded from the following approximation. The relationship approximated by the blue solid line describes Paris law behavior, which is insensitive to both $R$ and $R H$, and therefore corresponds to an FCG characteristic without an SCC contribution. In contrast, owing to the SCC contribution, the relationships at $0.02 \mathrm{~Hz}$ depend strongly on $R$ (as will be explained in a subsequent section). At $20 \mathrm{kHz}$ and $R H>90 \%$, very slow crack growth (i.e., growth rate: $\mathrm{d} a / \mathrm{d} N=10^{-13} \mathrm{~m} /$ cycle) is observed at $\Delta K<1.0 \mathrm{MPa} \sqrt{\mathrm{m}}$ of each alloy. This behavior is absent at $R H<20 \%$. For $R H<20 \%$, when the $\Delta K$ value increases to $1.0-1.5 \mathrm{MPa} \sqrt{\mathrm{m}}$, the crack begins to grow abruptly up to the FCG characteristic. This abrupt increase in $\mathrm{d} a / \mathrm{d} N$ occurs at lower $\Delta K$ values for $R H>90 \%$ (than the $\Delta K$ values associated with $R H<20 \%$ ) and the subsequent crack growth rate is higher than the FCG characteristic. Moreover, this behavior is more pronounced in the 6061 and 6082 alloys than in the 7075 alloy. Crack growth mechanism of 6061 will be discussed in section 3.2 for Fig. 6 .

Figure 3 shows the relationships between the timedependent crack growth rate, $\mathrm{d} a / \mathrm{d} t$, and $K_{\max }$ at $R=0.8$ and 0.9. The data obtained from the SCC test, where $\Delta K$ is

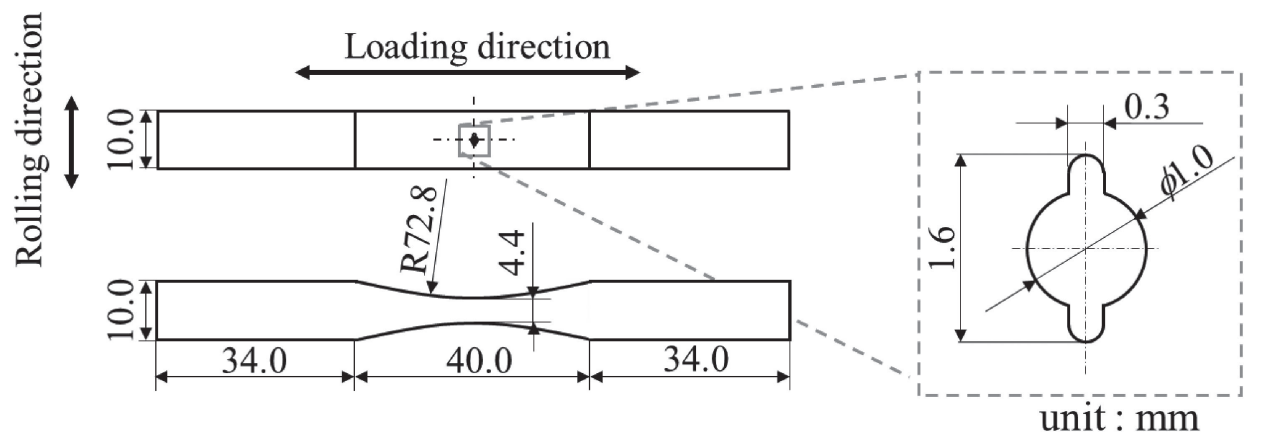

Fig. 1 Specimen geometry for ultrasonic fatigue crack growth test. The resonant frequency was adjusted to $\sim 20 \mathrm{kHz}$ by the length of the specimen. 


\begin{tabular}{|lclll|}
\hline 6061 & $0.02 \mathrm{~Hz}$ & $20 \mathrm{~Hz}$ & $20 \mathrm{kHz}$ & Solid symbol \\
$R=0.9$ & $\square$ & $\Delta \Delta$ & $\triangleright \triangleright$ & $R H>90 \%$ \\
$R=0.8$ & $\diamond$ & $\nabla \nabla$ & $\triangleleft \triangleleft$ & Open symbol \\
\hline
\end{tabular}

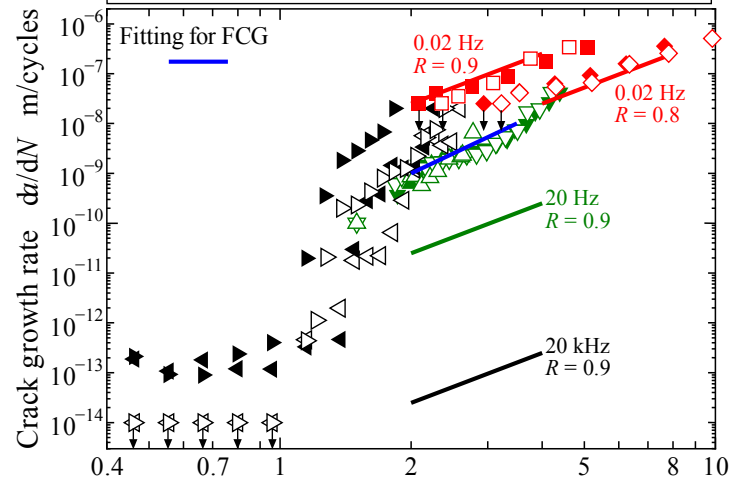

(a) Stress intensity factor range $\Delta K \mathrm{MPa} \sqrt{\mathrm{m}}$

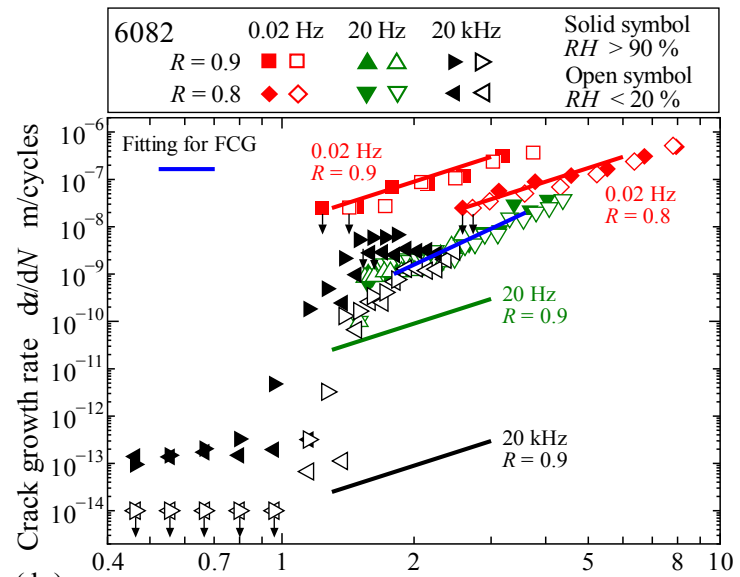

(b)

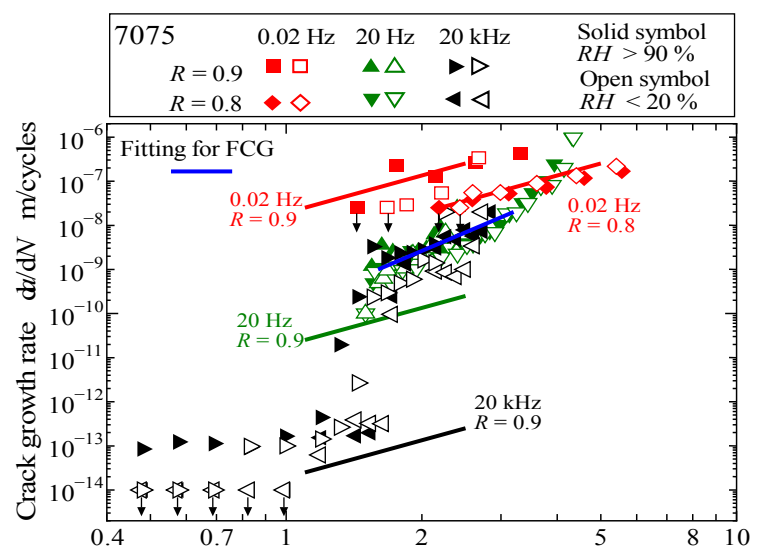

(c)

Stress intensity factor range $\Delta K \mathrm{MPa} \sqrt{\mathrm{m}}$

Fig. 2 Fatigue crack growth characteristics at $R=0.8$ and 0.9 , and the corresponding $\mathrm{d} a / \mathrm{d} N-\Delta K$ relationships for (a) 6061, (b) 6082, and (c) 7075. The lines (except for the blue one) correspond to values calculated from the SCC characteristics.

zero under the sustained loading of $K_{\max }$, are also shown. Red, green, and black lines denote the relationships between $\mathrm{d} a / \mathrm{d} t(=(\mathrm{d} a / \mathrm{d} N) f)$ and $K_{\max }(=\Delta K /(1-R))$ calculated from the FCG characteristics represented by the blue line in Fig. 2. For the FCG tests performed at $0.02 \mathrm{~Hz}$ of each alloy, the measured $\mathrm{d} a / \mathrm{d} t$ is considerably higher than the value calculated from the FCG characteristics, but is similar to that determined from the SCC test. This suggests that the crack growth is controlled by the SCC (rather than the FCG) mechanism. These experimental data are approximated by the

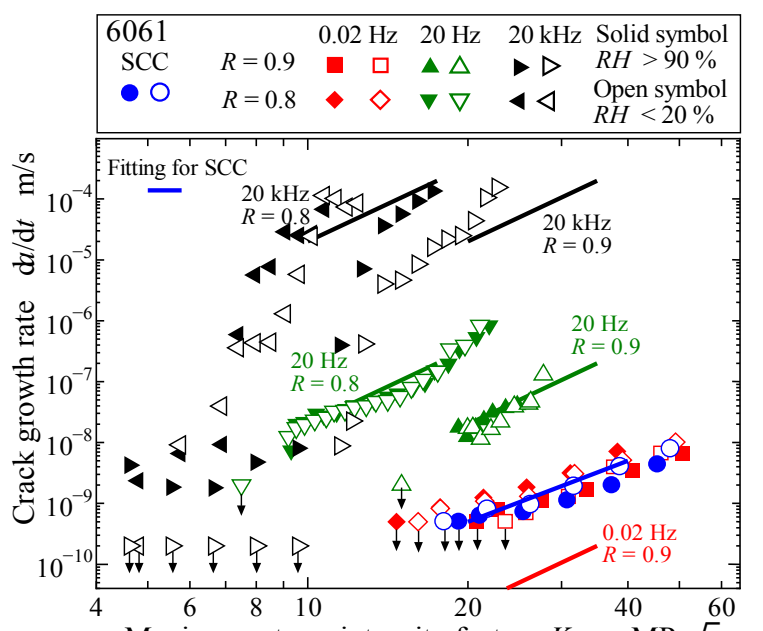

(a) Maximum stress intensity factor $K_{\max } \mathrm{MPa} \sqrt{\mathrm{m}}$

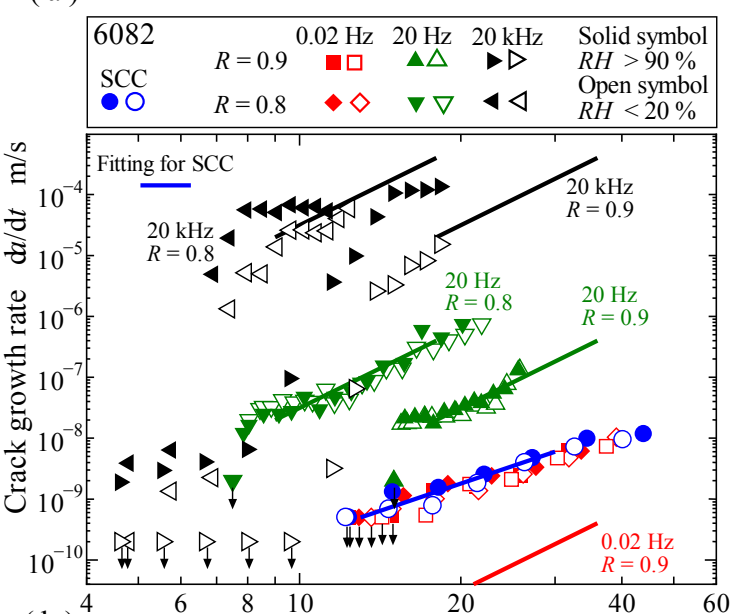

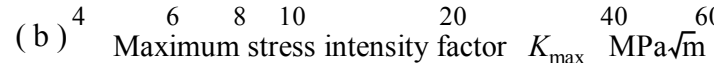

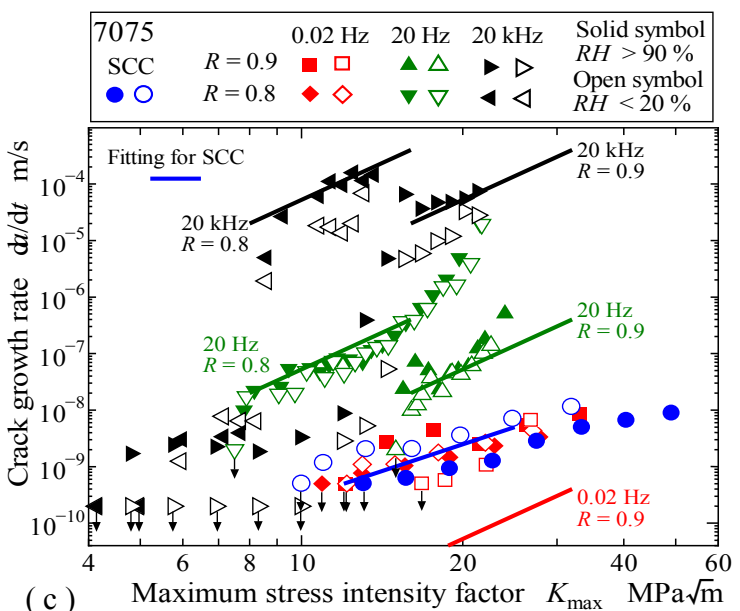

Fig. 3 Time-dependent crack growth characteristics at $R=0.8$ and 0.9 , and the corresponding $\mathrm{d} a / \mathrm{d} t-K_{\max }$ relationships for (a) 6061, (b) 6082, and (c) 7075, where the lines (except for the blue one) correspond to values calculated from the FCG characteristics.

blue solid line corresponding to SCC characteristics. The red, green, and black lines in Fig. 2 denote the relationships between $\mathrm{d} a / \mathrm{d} N(=(\mathrm{d} a / \mathrm{d} t) / f)$ and $\Delta K\left(=K_{\max }(1-R)\right)$, determined from the SCC characteristics. For the FCG tests at $20 \mathrm{~Hz}$ and $20 \mathrm{kHz}$, the calculated $\mathrm{d} a / \mathrm{d} N$ is considerably lower than the measured value, indicating that the SCC contributions are negligible. For the same conditions, Fig. 3 shows that the measured $\mathrm{d} a / \mathrm{d} t$ values are higher than those 
associated with the SCC characteristics, but concur with the values calculated from the FCG characteristics. This behavior indicates that the crack growth is controlled by the FCG (rather than the SCC) mechanism. However, typical crack growth behavior at $20 \mathrm{kHz}$ includes acceleration based on the FCG characteristics at $R=0.9$ and very slow crack growth at $\Delta K<1.0 \mathrm{MPa} \sqrt{\mathrm{m}}$. This slow growth occurs at $R H>90 \%$, but is absent at $R H<20 \%$.

Although crack growth at $0.02 \mathrm{~Hz}$ is controlled by the SCC mechanism, the FCG contribution may increase when the cyclic component of loading is increased. Figure 4 presents the relationships between $\mathrm{d} a / \mathrm{d} N$ and $\Delta K$ at $R=0.5$ as well as the data obtained for $0.02 \mathrm{~Hz}, R=0.8$, and 0.9 , previously shown in Fig. 2. Depending on the specimen geometry and loading frequency, the data at $R=0.5$ for $0.02 \mathrm{~Hz}, 20 \mathrm{~Hz}$, and $20 \mathrm{kHz}$ exhibit some scatter. However, these values are described by a unique Paris law relationship corresponding predominantly to the FCG mechanism, as the $\mathrm{d} a / \mathrm{d} N$ values calculated from the SCC characteristics are lower than the measured values (see Fig. 4). When the cyclic component of the loading at $0.02 \mathrm{~Hz}$ is reduced at $R=0.8$ and 0.9 , the measured and the calculated $\mathrm{d} a / \mathrm{d} N$ values increase more than the $\mathrm{d} a / \mathrm{d} N$ measured at $R=0.5$. This results from the SCC contribution, as indicated by the fracture surface discussed in the subsequent section. These results also suggest that the crack growth behavior is controlled by either the SCC or the FCG mechanism with no mutual interaction.

\subsection{Fracture surface observation}

Scanning electron micrographs (see Fig. 5) are obtained for the 6061 samples subjected to FCG testing at $R=0.5$, 0.8 , and 0.9 . These micrographs show the boundary region between the precrack regions, i.e., intergranular fatigue fracture surfaces (see upper part of the micrographs), and the early crack growth regions formed during testing. The crack growth behavior is dominated by FCG and, hence, FCG-characteristic morphology is observed for the test performed at $R=0.5$. However, a dimpled fracture surface is observed for FCG tests performed at $R=0.8$ and 0.9 . The surface morphology occurring for 6082 is similar to that observed for 6061. Nevertheless, a different morphology is observed for 7075 , i.e., delamination (vertical cracks perpendicular to the macroscopic fracture surface) occurs when the crack growth behavior is dominated by SCC. Our previous work revealed that 6061 and 6082 specimens subjected to SCC tests exhibit a dimpled fracture surface, whereas the 7075 specimens undergo delamination. ${ }^{10)}$ Consistent with the results shown in section 3.1, the crack growth behavior is controlled by either FCG or SCC mechanisms with no mutual interaction, as stipulated in the traditional model developed by McEvily and Wei. ${ }^{7)}$

Figure 6 compares the fracture surfaces of 6061 tested at $R=0.9$ and $f=20 \mathrm{kHz}$ for (a) $R H>90 \%$ and (b) $R H<$ $20 \%$, where the $\mathrm{d} a / \mathrm{d} N$ corresponding to a $\Delta K$ value varies as shown in Fig. 2. For $R H>90 \%$, very slow FCG is observed, although the $\Delta a$ is $<100 \mu \mathrm{m}$ from the boundary between the precrack and the test region. An intergranular faceted fracture surface occurs for both $R H$ conditions, but the facets formed at $R H>90 \%$ are smaller than those formed at $R H<20 \%$. The basis for the influence of $R H$ on the facet size remains

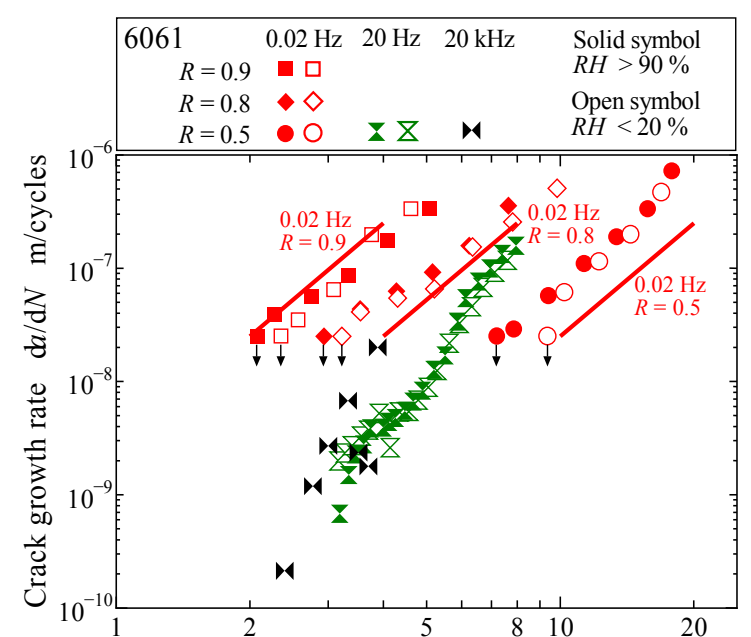

(a) Stress intensity factor range $\Delta K \mathrm{MPa} \sqrt{\mathrm{m}}$
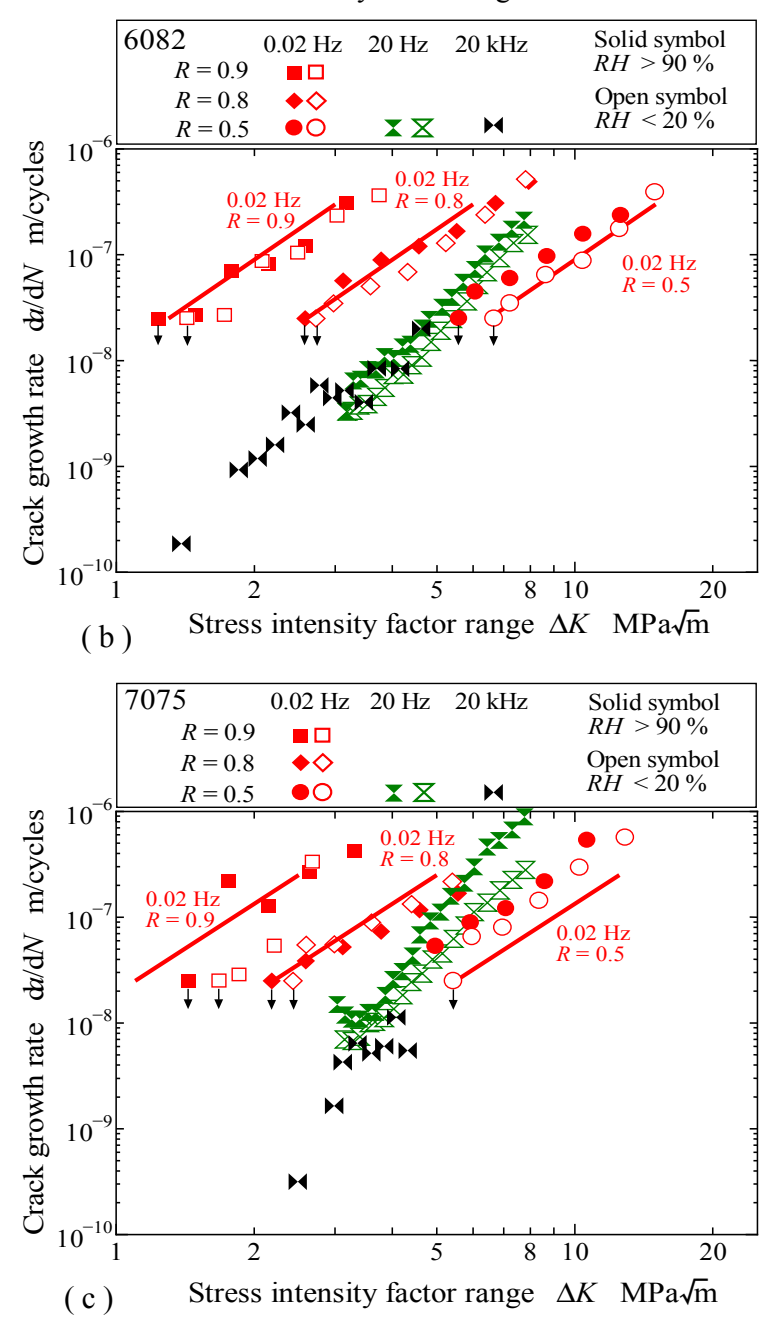

Fig. 4 Fatigue crack growth characteristics at $R=0.5$ and the corresponding $\mathrm{d} a / \mathrm{d} N-\Delta K$ relationships for (a) 6061, (b) 6082, and (c) 7075. The lines correspond to values calculated from the SCC characteristics.

unclear. However, dislocation movement may have played an important role in this behavior (our preliminary calculations of the hydrogen diffusion and the dislocation velocity revealed that the hydrogen could not catch up with the dislocation movement during cyclic loading at $20 \mathrm{kHz}$ ). As discussed in the Introduction, differences in $R H$ lead to changes in the hydrogen conditions at the crack tip. Further 


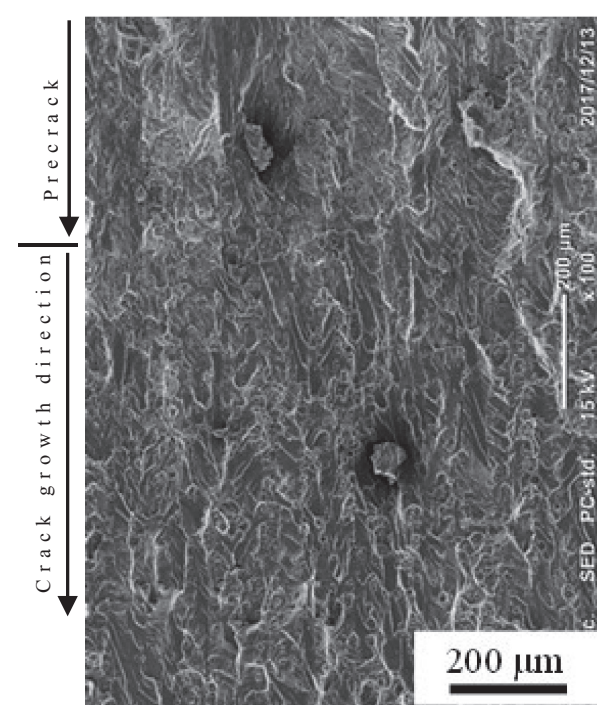

( a ) $\quad R=0.5$

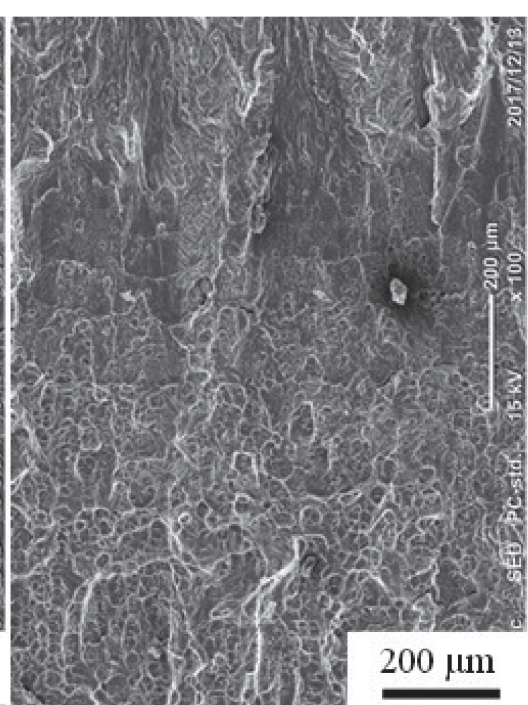

(b) $\quad R=0.8$

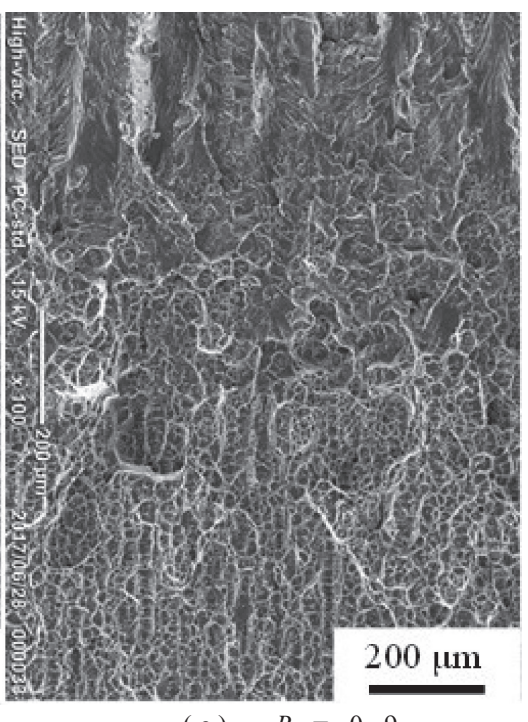

(c) $\quad R=0.9$

Fig. 5 SEM micrographs showing the fracture surfaces of 6061 tested at $f=0.02 \mathrm{~Hz}$ and $R H>90 \%$.

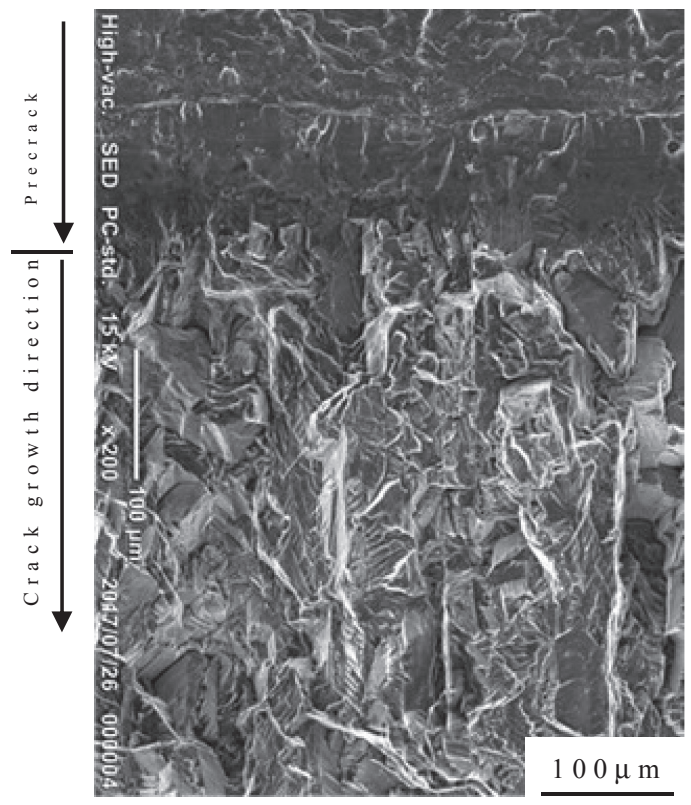

( a ) $\quad$ RH $>90 \%$

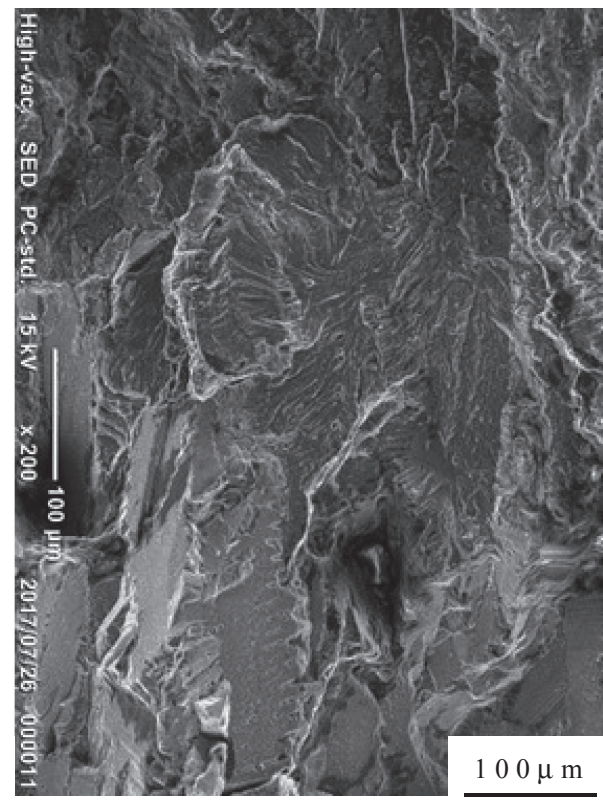

(b) $\quad$ RH $<20 \%$

Fig. 6 SEM micrographs showing the fracture surfaces of 6061 tested at $R=0.9$ and $f=20 \mathrm{kHz}$.

investigation is required to determine the basis for an increase in the FCG rate at $20 \mathrm{kHz}$ and $R H>90 \%$.

\subsection{Very slow fatigue crack growth (FCG)}

The very slow FCG observed in the FCG tests performed at $R H>90 \%$ occurs under different test conditions, e.g., long-term FCG tests performed at $K_{\max }=5 \mathrm{MPa} \sqrt{\mathrm{m}}$ constant condition, $R H>90 \%$, and $20 \mathrm{kHz}$. The test is also conducted at $R H<20 \%$. In Ref. 6), the aluminum alloys used in these tests (i.e., 6061, 6066, and 6351) are referred to as finegrained (FG) materials of lot (2). The $N$ dependence of $a$ and $\Delta K$ associated with 6061 is shown in Fig. 7. The load range, $\Delta P$, is set such that $R$ is initially 0.9 and then increases by $20 \%$ at every $N=10^{9}$ cycles. When crack growth is detected, this growth is kept constant until the crack is arrested or $a$ increases abruptly. This abrupt increase in $a$ results in specimen fracture and occurs at $N$ of $14.5 \times 10^{9}$ or $9.2 \times 10^{9}$ (see Fig. 7). These crack growth characteristics are described by the relationships between $\mathrm{d} a / \mathrm{d} N$ and $\Delta K$ shown in Fig. 8 and the results obtained for $R=0.5,0.8$, and 0.9 shown in Fig. 2(a) or Fig. 4(a). Consistent crack growth characteristics are confirmed for the $K_{\max }$ and $R$-constant test conditions.

Figures 9 and 10 show SEM micrographs of the samples tested at $R H>90 \%$ and $R H<20 \%$, respectively. The fracture surface boundary between the precrack and the region undergoing $K_{\max }$-constant FCG tests is shown in each case. In each case, the image shown in (b) represents a magnified view of the rectangle-enclosed region shown in (a). The tide marks observed on the precracks result from the intermittent loading, as confirmed by comparing the macroscopic crack growth rates. The fracture surface of the FCG 

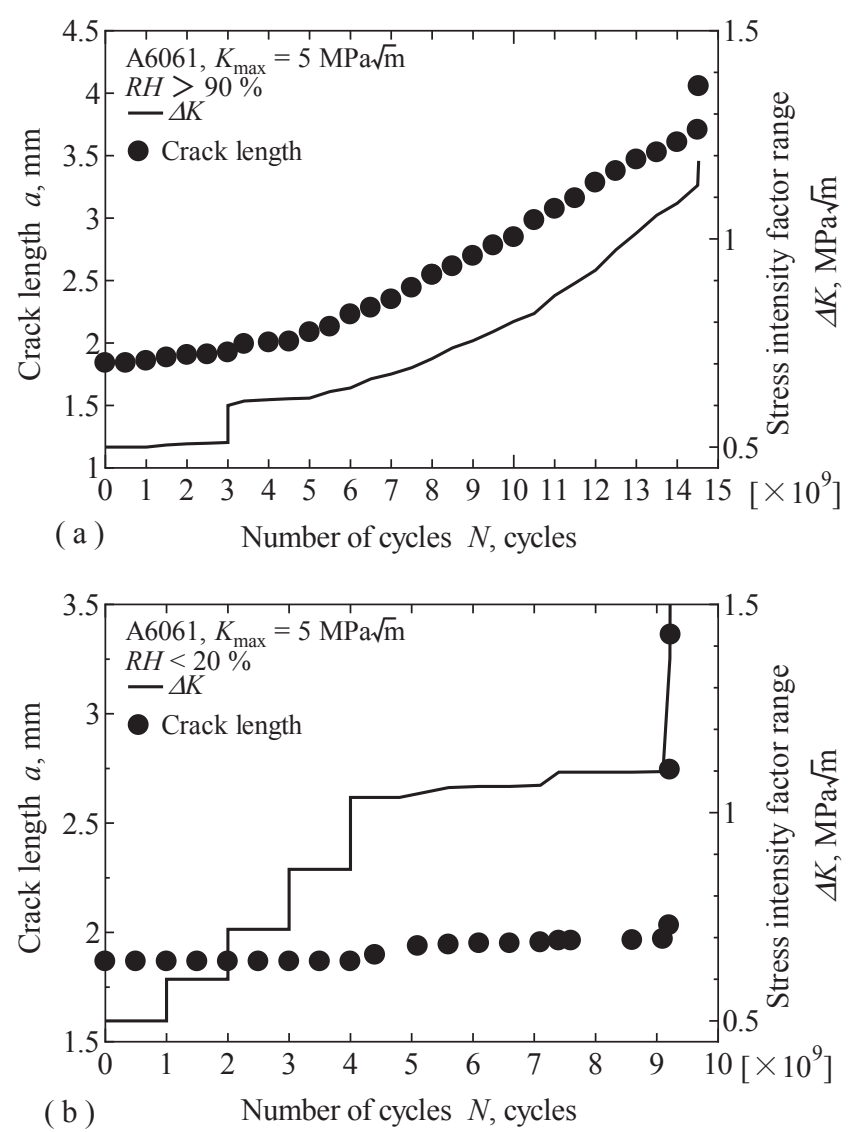

Fig. 7 Variations in $a$ and $\Delta K$ during long-term testing of 6061 subjected to a $K_{\max }=5 \mathrm{MPa} \sqrt{\mathrm{m}}$ constant condition and $20 \mathrm{kHz}$ for (a) $R H>90 \%$ and (b) $R H<20 \%$

test region differs significantly from the precrack region for both $R H$ conditions, but the $R H>90 \%$ surface is considerably flatter and smoother than the $R H<20 \%$ surface. Although the fracture surface was rinsed via ultrasonic cleaning prior to these SEM observations, some particles are observed on the flat fracture surface shown in Fig. 9(b). Prior to this cleaning, the surface was covered with aluminum oxide, as confirmed by an electron probe micro

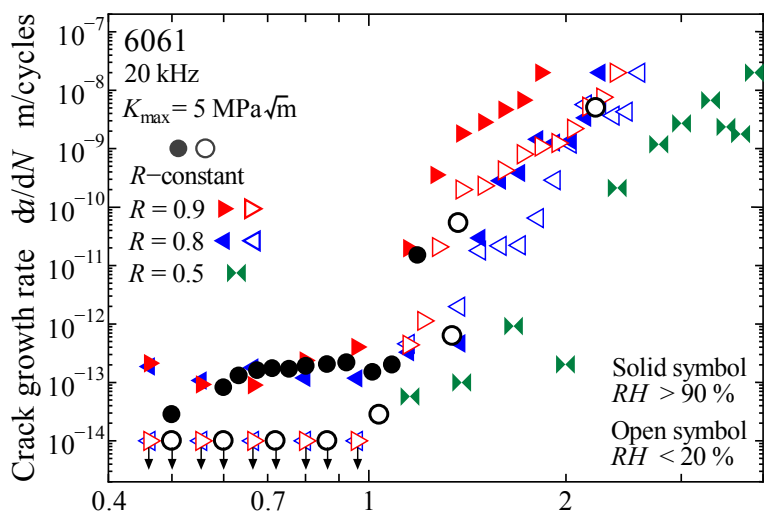

Stress intensity factor range $\Delta K \mathrm{MPa} \sqrt{\mathrm{m}}$

Fig. 8 FCG characteristics of the long-term test compared with those of the $R$-constant tests shown in Fig. 2(a) and Fig. 4(a).

analyzer (EPMA). Long-term FCG tests have confirmed that the fracture surface is covered with aluminum oxide (for crack growth occurring at a very slow rate) and this oxide can be removed via the blank replica technique. ${ }^{11)}$

Similar results are obtained for the other aluminum alloys (i.e., 6066 and 6351) exposed to the same test conditions, indicating that the very slow FCG behavior is insensitive to the chemical composition of the alloy. This is confirmed by the results of the $R$-constant tests (see Figs. 2 and 3). These results suggest that the mechanism governing very slow FCG is dominated by mechanical fatigue behavior aided by high relative humidity. Observations of the specimen surface indicate that some powders were formed near the crack tip during testing. When the relative humidity and the cyclic frequency are high, the moisture in air condenses to water at the crack tip. ${ }^{12)}$ Owing to the meniscus behavior of the water, aluminum oxide may accumulate at the crack tip and, hence, cyclic loading results in further damage to the passive oxide film near the tip. These results suggest that, due to the numerous cyclic loads, the very slow crack growth behavior in humid environments occurs via peeling-off of the oxide film near the crack tip.

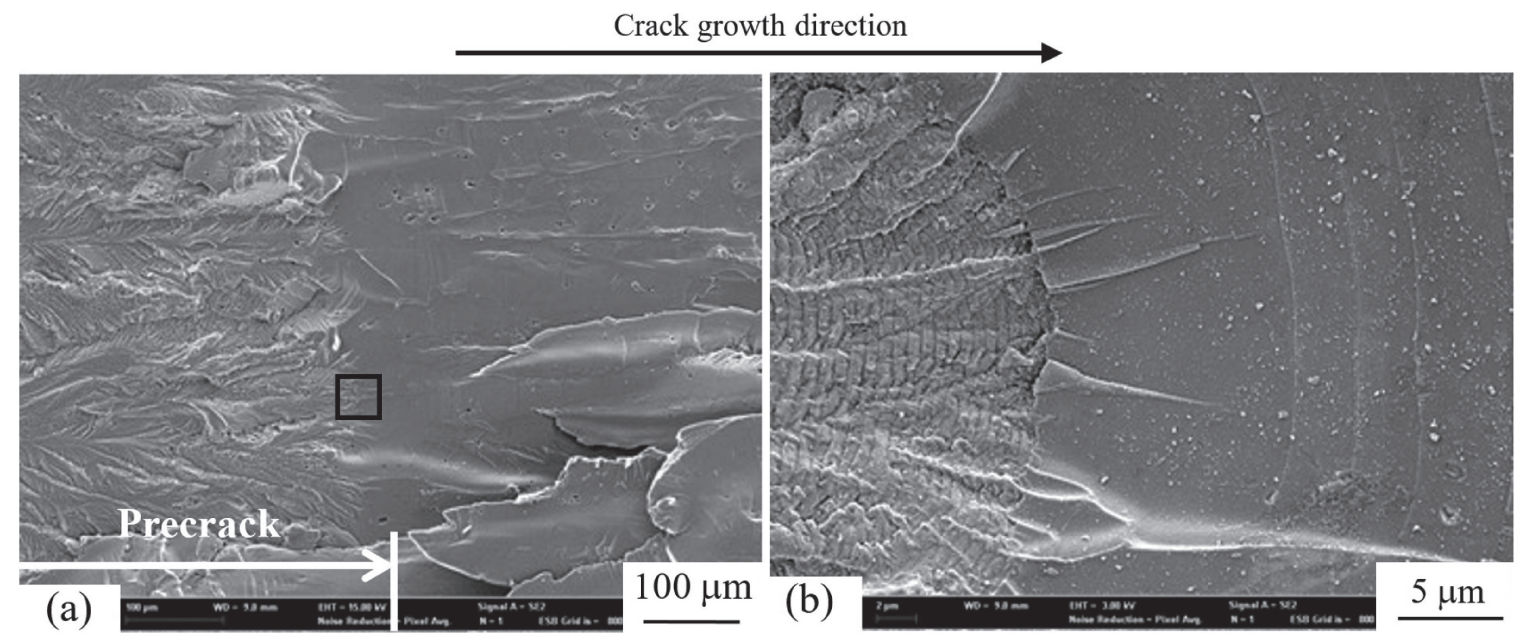

Fig. 9 SEM micrographs showing (a) the fracture surface of 6061 subjected to a $K_{\max }=5 \mathrm{MPa} \sqrt{\mathrm{m}}-\mathrm{constant}$ test at $20 \mathrm{kHz}$ and $R H>90 \%$ and (b) a magnified view of the region enclosed in the rectangle. 


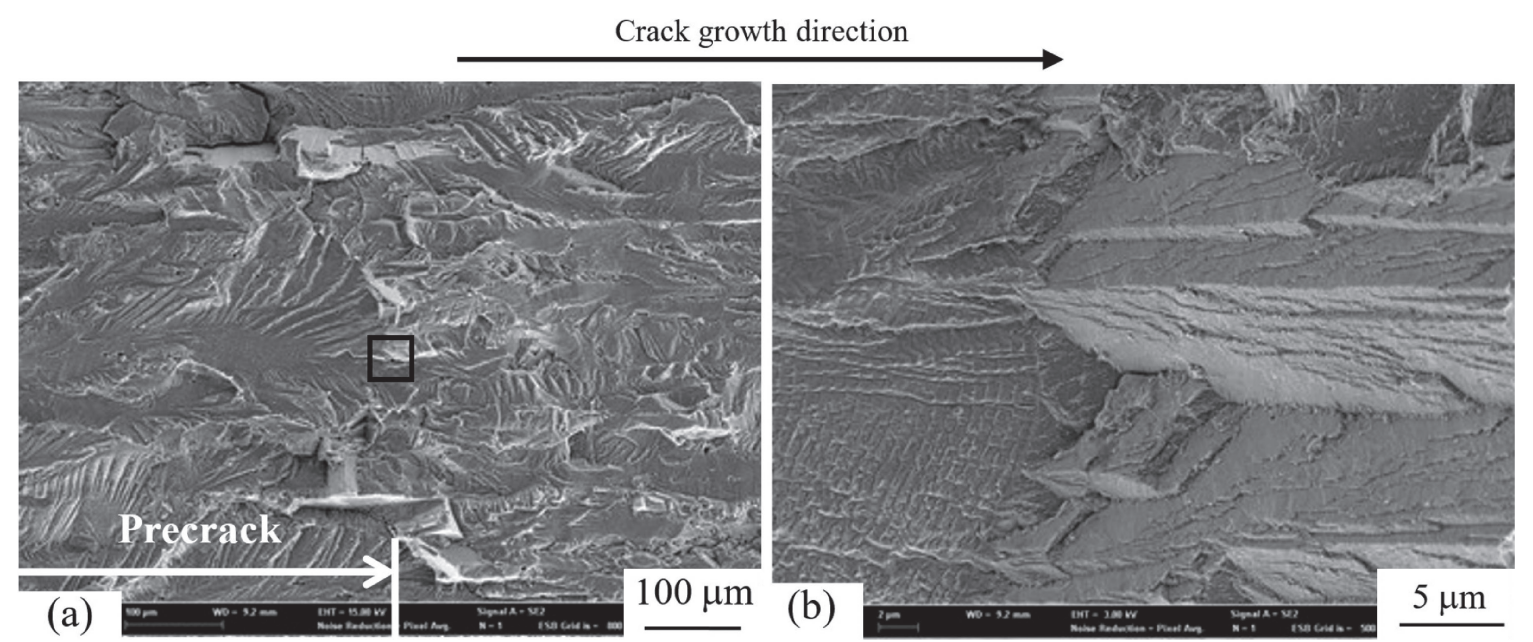

Fig. 10 SEM micrographs showing (a) the fracture surface of 6061 subjected to a $K_{\max }=5 \mathrm{MPa} \sqrt{\mathrm{m}}-\mathrm{constant}$ test at $20 \mathrm{kHz}$ and $R H<20 \%$ and (b) a magnified view of the region enclosed in the rectangle.

\section{Conclusions}

In the present study, crack growth characteristics are investigated for various aluminum alloys subjected to static and cyclic loading conditions in humid and dry air environments. The results obtained are summarized as follows.

(1) Crack growth tests and observations of the fracture surfaces reveal that the crack growth behavior is controlled by either the SCC or FCG mechanism with no mutual interaction. This is consistent with the traditional model proposed by McEvily and Wei.

(2) Regardless of the alloy and the stress ratio, a very slow crack growth rate (below $10^{-13} \mathrm{~m} /$ cycle) is realized for ultrasonic fatigue tests performed at $20 \mathrm{kHz}$ in humid air. Furthermore, SEM observations suggest that, owing to the numerous cyclic loads, this growth behavior occurs via the peeling-off of the oxide film near the crack tip.

\section{Acknowledgment}

This work was supported in part by the Ministry of Education, Science, Sports and Culture, Grant-in-Aid for Scientific Research (C) No. 15K05686. We thank Liwen
Bianji, Edanz Editing China (www.liwenbianji.cn/ac), for editing the English text of a draft of this manuscript.

\section{REFERENCES}

1) G. Itoh: Zairyo-to-Kankyo 65 (2016) 432-437.

2) High Pressure Institute of Japan: HPIS E 103:2018, (2018) pp. 1-14.

3) N. Kawagoishi, K. Kariya, Y. Nakamura, T. Nagano and R. Iwamoto: Trans. Jpn. Soc. Mech. Eng. 83 (2017) 17-00274.

4) K. Endo, K. Komai and K. Minoshima: J. Soc. Mater. Sci. Jpn. 31 (1982) 822-827.

5) K. Komai and K. Minoshima: Trans. Jpn. Soc. Mech. Eng. A 50 (1984) 1804-1810.

6) T. Ogawa, S. Hasunuma, S. Watanabe, N. Sogawa, T. Kanezaki, S. Mano and K. Miyagawa: J. High Press. Inst. Jpn. 54 (2016) 277-288.

7) A.J. McEvily and R.P. Wei: Corrosion Fatigue: Chemistry, Mechanics and Microstructure, (National Association of Corrosion Engineers, 1972) pp. 381-395.

8) T. Ogawa, S. Hasunuma, N. Sogawa, D. Yoshida, T. Kanezaki and S. Mano: Proc. ASME 2014 Press. Vessels \& Pip. Conf., (2014) PVP2014-28236.

9) K. Sakurai, Y. Miyai, S. Hasunuma, T. Ogawa, M. Woo and M. Takanashi: J. Soc. Mater. Sci. Jpn. 66 (2017) 879-886.

10) T. Ogawa, S. Hasunuma, T. Shirawachi and N. Fukada: J. High Press. Inst. Jpn. 57 (2018) 24-33.

11) S. Suzuki, S. Hasunuma, T. Ogawa, S. Mano and K. Miyagawa: Proc. Mater. Mech. Conf. 2017, (2017) OS0541.

12) T. Ogawa, K. Tokaji and S. Ochi: Trans. Jpn. Soc. Mech. Eng. 52 (1986) 399-405. 